\title{
Estudio descriptivo de corte transversal para valorar las experiencias y expectativas de estudiantes de la licenciatura en Enfermería de la Universidad Católica Argentina, en relación a la investigación
}

\author{
Descriptive cross-sectional study to assess the \\ experiences and expectations with students of the \\ degree in Nursing at the Catholic University of \\ Argentina, in relation to research
}

René Toconás', Araceli Alesio², María B. Delgado³, Julieta Ferrufino², Alexander Cherrepano4, Karina Garay², Narda De La Cruz $^{6}$, Romina Martínez ${ }^{2}$, Lucas Hoquigaray ${ }^{5}$

\section{RESUMEN}

Objetivo. Describir la importancia de la investigación en estudiantes de la Licenciatura de enfermería de la Universidad Católica Argentina en el año 2018. Material y método. Estudio transversal mediante una encuesta autoadministrada realizada a alumnos de la Universidad Católica de la Licenciatura de Enfermería. Se incluyeron alumnos matriculados en la asignatura Metodología en Investigación; se excluyeron las encuestas incompletas.

Resultados. El 42\% de los alumnos había recibido formación en Metodología de Investigación previamente, lo cual les permitió ser partícipes en algún estudio. Si bien solo un $41.6 \%$ presentó interés por el desarrollo de la asignatura; ninguno de los encuestados consideró que asistía por obligación. Del total de los encuestados solo el $8 \%$ participó alguna vez en un trabajo de investigación de su hospital. El porcentaje de participación como autor en trabajos de investigación publicados fue menor a un $25 \%(p<0.001)$. El objetivo de este trabajo es describir los hallazgos de la encuesta y resaltar la importancia de motivar al alumnado en etapa de formación académica.

Conclusión. En concordancia con investigaciones anteriores publicadas, nuestro trabajo contribuye a destacar la importancia de la investigación para la enfermería y la implementación de la metodología en investigación durante el proceso de formación del profesional. La enfermería ha evolucionado acompañando a los avances de la tecnología en salud y también en la práctica asistencial. Fomentar el desarrollo de los trabajos de investigación tanto autónomos como en equipos multidisciplinarios mejoraría la labor diaria de los enfermeros.

\section{ABSTRACT}

Objective. To describe the importance of research in students of the course of Bachelor in Nursing of the Catholic University of Argentina in the year 2018.

Material and methods. Cross-sectional study through a self-administered multiple-choice survey to students of the Bachelor of nursing at the Catholic University. Students enrolled in Research Methodology of the current year were included, and those who did not complete the survey were excluded.

Results. $42 \%$ of the students had previously received training in research, which allowed them to participate in such studies. Only $41.6 \%$ showed interest in the development of the subject of Research Methodology. None of the respondents considered that they were obliged to attend. Of all the respondents, only $8 \%$ ever participated in a research project at their hospital. The percentage of participation as an author in published research papers was less than $25 \%(p>$ $0.00 \mathrm{I}$ ). The object of this paper is to describe the findings of this inquiry and to stress the importance of motivating students at this level of their training. Conclusion. In agreement with previously published research, our paper stresses the importance of research in nursing and the implementation of research in the professional training process. Nursing has evolved accompanying advances in health technology and also in delivery of care. Fomenting the development of papers in research, both individually and in teams, will improve nursing in their day-to-day practice.

Keywords: research, nursing, methodology.

Palabras clave: investigación, enfermería, metodología.

I. Servicio de Enfermería,Terapia Intensiva de Pediatría. Sanatorio Sagrado Corazón. Buenos Aires, CABA.

2. Servicio de Enfermería. Internación general. Hospital Británico de Buenos Aires, CABA.

3. Enfermería Domiciliaria.

4. Servicio Enfermería, Psiquiatría. Sanatorio San José. Buenos Aires, CABA.

5. Servicio de Enfermería. Terapia Intensiva. Hospital Británico de Buenos Aires, CABA.

6. Servicio de Enfermería de Guardia Pediátrica. Sanatorio Sagrado Corazón. Buenos Aires, CABA.

Correspondencia: Enfermero Lucas Hoquigaray. Servicio de Enfermería, Terapia Intensiva, Hospital Británico de Buenos Aires. Perdriel 74,

CI280AEB CABA, Rep.Argentina.Tel: 43096400 int 1257. Email: lucasjh1978@gmail.com

Los autores declaran no poseer conflictos de intereses.

Recibido: 25/6/2018 |Aceptado: 20/7/2018 


\section{INTRODUCCIÓN}

La educación universitaria en Enfermería actualmente enfrenta desafíos tales como la generación de nuevos conocimientos, fortalecimiento de capacidades éticas, cognitivas, disciplinarias, humanísticas y tecnológicas. La palabra investigación significa "buscar de nuevo" o "estudiar con cuidado" . Polit y Francisca Hernández de Canales describieron que la "investigación científica en ciencias de la salud” está diseñada para ampliar la base de conocimientos o formular teorías mediante un proceso controlado, empírico, reflexivo y crítico ${ }^{2,3}$.

Según la OMS, los enfermeros necesitan investigar para contribuir a que la población alcance estilos de vida saludables mediante una atención adecuada, y que es responsabilidad de esta área, procurar los conocimientos necesarios para poder hacerlo. Por lo tanto, la meta final de todo profesional de enfermería es mejorar la práctica, para que los servicios brindados a la sociedad tengan la mayor eficacia y eficiencia posibles ${ }^{4}$. La organización curricular debería permitir desarrollar en los estudiantes una visión integral de los múltiples factores que interactúan en la dinámica del proceso salud-enfermedad para lograr una mejor comprensión e intervención con el objeto de estudio (cuidado de la salud humana) y dar sentido a la práctica profesional, desde una perspectiva humanista y sostenible ${ }^{5}$.

Se ha descrito previamente según la Asociación Nacional de Universidades e Instituciones de Educación Superior (ANUIES) que, para asegurar la calidad educativa, es necesario conocer la congruencia que existe, entre el perfil de egreso y las exigencias del ejercicio profesional ${ }^{6}$. Conocer la satisfacción de los egresados en relación con los contenidos curriculares obtenidos durante su proceso formativo, permitirá sustentar la actualización y/o la adecuación de los planes y programas de estudios.

Numerosos autores han expresado su opinión sobre la carrera de grado y postgrado de Enfermería, coincidiendo en que la formación profesional, desde el nivel inicial o pregrado debe motivar a la evaluación permanente mediante la lectura crítica, el manejo de la información en la búsqueda con bases de datos, el trabajo en equipo y el manejo de evidencias científicas; y de esa manera tener promover la formación de las nuevas generaciones de enfermeros ${ }^{7-11}$.

La investigación constituye una actividad esencial para los profesionales de enfermería. Aunque ha habido un incremento en los últimos años, no está exenta de dificultades y limitaciones que obstaculizan dicha actividad. Podemos mencionar la falta de tradición en esta área, la variabilidad de la formación entre las diferentes escuelas de enfermería, el déficit de formación en aspectos metodológicos y estadísticos. Es por ello, la necesidad de insistir en formación en metodología para apoyar la realización de estudios de investigación en enfermería y/o multidisciplinarios, para mejorar los servicios de salud, y poder diferenciar que los
No estoy seguro En desacuerdo De acuerdo
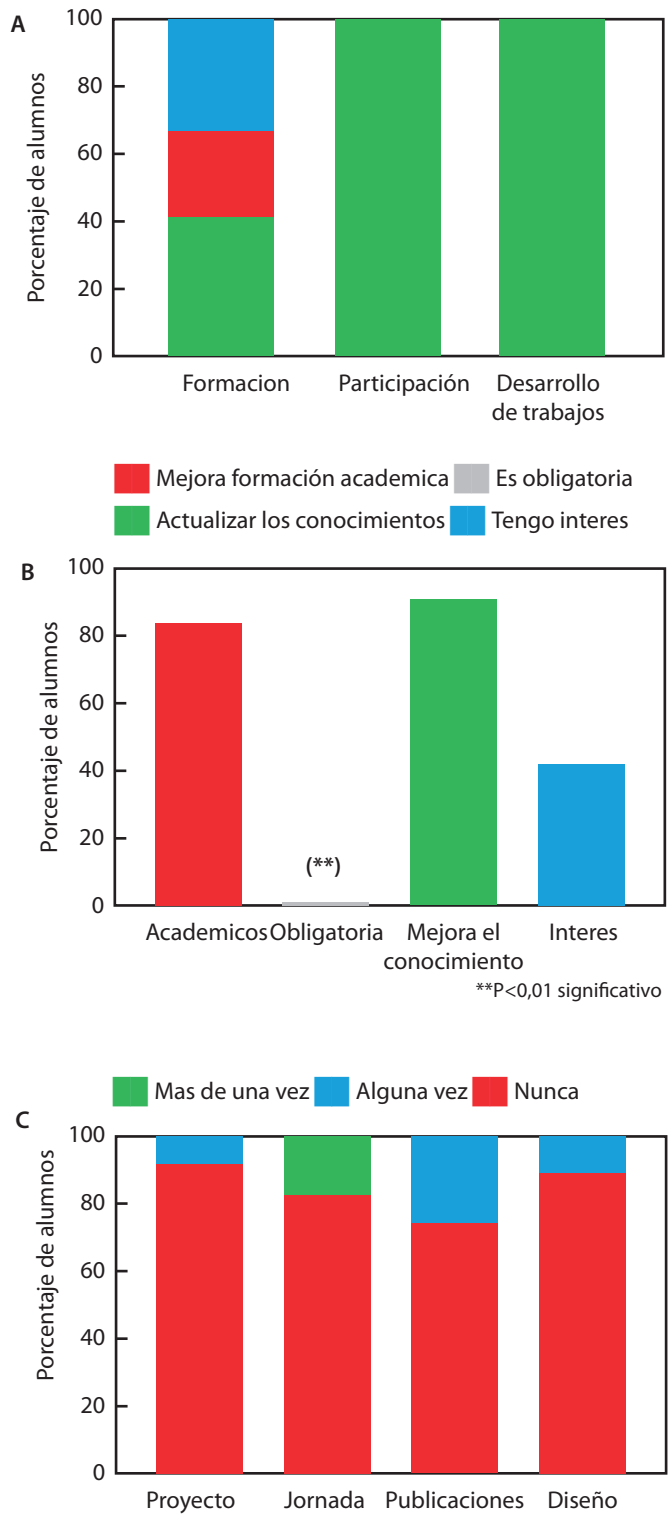

Figura I. Resultados de las respuestas de la encuesta. (I.A) Formación, participación e interés sobre metodología de la investigación en estudiantes de Licenciatura en Enfermería de la Universidad Católica Argentina. (I.B) Motivación de los alumnos de la Licenciatura en Enfermería en la Universidad Católica Argentina. (I.C) Experiencia y participación de los alumnos de la Licenciatura en Enfermería de la Universidad Católica Argentina en relación a trabajos de investigación.

planes de cuidados sean más sólidos, basándose en la evidencia y no en la experiencia intuitiva en dicha área.

En función de esto, se ha desarrollado esta encuesta para estimar la proporción de estudiantes que consideran importante la materia metodología en investigación en este punto de su formación y valorar los antecedentes del mismo.

\section{MATERIALESY MÉTODOS}

\section{Diseño del estudio}

Se realizó un estudio exploratorio transversal. Se utilizó una encuesta anónima, (adjunta encuesta en Anexo 
Tabla I. Resultados acerca de las expectativas para realizar trabajos de investigación.

\begin{tabular}{|l|c|c|c|}
\hline Pregunta I & $\begin{array}{c}\text { De } \\
\text { acuerdo }\end{array}$ & $\begin{array}{c}\text { En } \\
\text { desacuerdo }\end{array}$ & $\begin{array}{c}\text { No estoy } \\
\text { seguro }\end{array}$ \\
\hline Formación & $42 \%$ & $25 \%$ & $33 \%$ \\
\hline $\begin{array}{l}\text { Participación importante para } \\
\text { la formación profesional. }\end{array}$ & $100 \%$ & $0 \%$ & $0 \%$ \\
\hline Mejora la labor diaria. & $100 \%$ & $0 \%$ & $0 \%$ \\
\hline
\end{tabular}

1), con preguntas cerradas a alumnos que se encontraban cursando el primer año de la Licenciatura de Enfermería durante el primer cuatrimestre del año 2018.

\section{Población}

Se incluyeron en este estudio a los alumnos que se encontraban cursando la Licenciatura de enfermería de 1er. año en la Universidad Católica Argentina. Enfermeras/os estudiantes matriculados, que asistieron a una clase de tipo presencial y que aceptaron participar en el estudio.

Se excluyeron a enfermeras/os que no asistieron a clase el día de recolección de datos y no completaron el instrumento de estudio.

\section{Variables registradas}

Se interrogó al alumno, se observó en función de la experiencia de su práctica diaria, si consideraba necesario la metodología de investigación en su formación académica y su participación.

La percepción de la importancia en la investigación valorada por la escala de Likert (de acuerdo, desacuerdo y no estoy seguro).

\section{Análisis estadísticos}

Los resultados se analizaron utilizando el software GraphPadPrism 7.04. Para la estadística se informaron la media y el intervalo de confianza. Para verificar si hubo diferencias significativas se utilizó el método de test de Kruskal-Wallis. Se consideró como significativa $\mathrm{p}<0.05$.

\section{RESULTADOS}

Los hallazgos de este estudio mostraron que el 100\% de los encuestados reconoció que cursar la materia Metodología de Investigación mejoraría el desarrollo profesional y laboral (Tabla 1). Se observó que el 100\% de los alumnos estuvo de acuerdo con la importancia de su participación en estudios de investigación para su desarrollo profesional (Tabla 1). Solo el 33\% no había recibido, previo a la instancia de la Licenciatura, formación académica que le permitiera participar en trabajos de investigación, aunque los resultados no fueron significativos (Figura 1A).

El 91.6\% consideró que debe actualizar sus conocimientos sobre el desarrollo en trabajos de investigación (Tabla 3).
Tabla 2. Resultados acerca de la experiencia para realizar trabajos de investigación.

\begin{tabular}{|l|c|c|c|}
\hline Pregunta 2 & Nunca & Alguna vez & Más de una vez \\
\hline Si ha participado & $92 \%$ & $8 \%$ & $0 \%$ \\
\hline Presencia en congresos & $83,3 \%$ & $0 \%$ & $16,7 \%$ \\
\hline $\begin{array}{l}\text { Participación como autor } \\
\text { de trabajos }\end{array}$ & $75 \%$ & $25 \%$ & $0 \%$ \\
\hline $\begin{array}{l}\text { Diseño de investigación } \\
\text { por iniciativa personal }\end{array}$ & $90,1 \%$ & $9,9 \%$ & $0 \%$ \\
\hline
\end{tabular}

Un $83.3 \%$ de los alumnos refirió que el curso mejoraría su formación académica, pero solo un $41.6 \%$ estuvo interesado en los temas a desarrollar durante la cursada. Cabe destacar que ningún alumno consideró que debe realizar el curso de forma obligatoria (Figura 1B).

Se percibió un incremento estadísticamente significativo de estudiantes que refirieron no haber tenido participación alguna en trabajos de investigación $(92 \%) \mathrm{p}<0.01$. Más aún, los resultados de la encuesta mostraron que el $83,3 \%$ no participó en jornadas o congresos y sólo un $16.7 \%$ participó más de una vez (Tabla 2).

Por otro lado, el 90.1\% nunca diseñó un estudio de investigación por iniciativa personal, solo un $9.9 \%$ alguna vez lo hizo (Figura 1C).

\section{DISCUSIÓN}

En las instituciones formadoras de recursos en salud, en sus diferentes niveles de educación superior, en los últimos años se ha promovido el desarrollo de cursos y concursos de investigación. A pesar de los esfuerzos desplegados por las diferentes instituciones en enfermería, estos han sido poco difundidos y socializados por la comunidad científica, lo cual da como resultado el poco desarrollo de la investigación en esta profesión.

La evolución de la profesión de enfermería obliga a establecer normas que se basen en la evidencia científica, ya sean en forma de guías de práctica clínica, planes de cuidados, algoritmos, etc., desechando ideas que están, por el peso de la tradición, firmemente arraigadas y permitiendo así, al profesional enfermero, una práctica adecuada y actualizada. Teniendo en cuenta que todo profesional de enfermería es consumidor crítico de investigación, productor potencial de investigación y transmisor del conocimiento, corresponde a todos los enfermeros impulsar el desarrollo de la investigación tomando conciencia de la importancia que esta tiene y los beneficios que aporta para el desarrollo disciplinario y profesional de la enfermería ${ }^{12,13}$.

Investigaciones realizadas en América Latina afirman que los artículos publicados por enfermería proceden en su mayoría de instituciones académicas, demostrando que esto puede deberse a que la investigación sea un requisito académico de interés institucional ${ }^{14,15}$. Esta información no concuerda con nuestro trabajo de investigación ya que se pudo apreciar que menos del $50 \%$ tuvo metodología como asignatura institucional. Es de des- 
Tabla 3. Resultados acerca de las necesidades para realizar trabajos de investigación.

\begin{tabular}{|l|c|}
\hline Pregunta 3 & Respecto al curso \\
\hline Mejora la formación académica & $100 \%$ \\
\hline Realización obligatoria & $0 \%$ \\
\hline $\begin{array}{l}\text { Considero que debo mejorar el co- } \\
\text { nocimiento }\end{array}$ & $91.6 \%$ \\
\hline Me interesa el tema & $41.6 \%$ \\
\hline
\end{tabular}

tacar la diferencia curricular de la heterogénea formación académica de los alumnos de la licenciatura ya que no son egresados de una misma institución. Sin embargo; también se aprecia un reducido porcentaje en trabajos, presentaciones y proyectos de investigación realizados por iniciativa propia del alumno de su centro.

Rojas y Méndez han demostrado previamente que el aprendizaje en investigación formativa que se relaciona con la motivación entre estudiantes y docentes ${ }^{16}$. En acuerdo con dichos autores los resultados obtenidos en este trabajo mostraron que el $41.6 \%$ tuvo interés en el desarrollo de la asignatura metodología de la investigación. Más aún, nuestros hallazgos mostraron que el 0\% del alumnado consideró que asistía a la asignatura por ser obligatoria. La gran mayoría de los encuestados refirieron que deben mejorar sus conocimientos en investigación y también que esto va a mejorar su formación académica. De este modo se estableció que existe motivación por parte del alumnado.
Reportes previos publicados por Miguel et al. en 1994 concluyeron que "la mayoría de los estudiantes presentaron actitudes de indiferencia hacia el curso de investigación (55.6\%), en la segunda instancia los alumnos de 4to año presentan actitudes de aceptación (30\%) y los 5 to actitudes de rechazo $(30.3 \%)^{17}$. Cabrera-Enríquez en 2011 concluyó "que el nivel de conocimientos sobre la investigación es deficiente y la actitud hacia ella es inadecuada. Para cambiar esta situación, es prioritario hacer cambios curriculares que garanticen la incorporación de los estudiantes a la investigación desde el inicio de la carrera y desarrollar competencias en investigación en los últimos años de estudio"18.

A través de la investigación demostramos la evolución en la práctica asistencial ya que esta práctica puede contribuir a fomentar la autonomía profesional y el rol independiente, conseguir participación en equipos multidisciplinarios. La enseñanza y la investigación dentro de los programas académicos son importantes ya que ayudan al desarrollo del estudiante y egresado adquiriendo nuevos conocimientos a través de la investigación, publicaciones y herramientas. En los últimos años la enfermería ha progresado en su plan curricular manteniendo un acompañamiento al avance de las nuevas tecnologías en salud, más aún en la práctica asistencial.

\section{BIBLIOGRAFÍA}

I. Burns N, Susan K. Investigación de Enfermería .3 Ed. España: Elsevier, S.A.2005;3-4.

2. Polit F. Investigación científica en ciencias de la salud. Hungler, P. Bernadette. $6^{\circ}$ Ed. México. D.F: Interamericana S.A. 2000;70I.

3. Hernández De Canales F. Metodología de la investigación. Suárez de Sarmiento B. Importancia de la investigación para el desarrollo de la disciplina de enfermería. Universidad de La Sabana, Colombia. Aquichan 2003;3(3):5-8.

4. Ruiz MT, Romá MT, Cartagena E. La función de investigación en enfermería. Enfermería Científica; 1990; I 05: | 6-21.

5. Facultad de Ciencias de la Salud. Plan de estudios de la Licenciatura en Enfermería. Tlaxcala: Universidad Autónoma de Tlaxcala;2007.

6. Ochoa S. A propósito de la enfermería basada en la evidencia: algunos cuestionamientos, limitaciones y recomendaciones para su implementación. Rev. Investigación Educación Enfermería 2005; 23(2): 138-46.

7. Marqués S. La Lectura crítica. Fundamental en Enfermería y para las Enfermeras. Evidencia 2009;6(27).

8. Ochoa S. A propósito de la enfermería basada en la evidencia: algunos cuestionamientos, limitaciones y recomendaciones para su implementación. Rev. Investigación Educación Enfermería 2005; 23(2): 138-46.

9. Lora López P. Reflexiones sobre el grado y postgrado de Enfermería: la investigación en Enfermería. RevIndexEnferm 2010;17(2):85-6.
10. Santos Heredero FX,RodríguezArias CA.Metodología Básica de investigación en Enfermería. I ${ }^{\circ}$ Ed. España:Díaz de Los Santos,S.A.2003;| I -2.

I I. Guevara Valtier MC, Cárdenas Villarreal VM, Hernández Cortés PL.Protocolos de Investigación de Enfermería. $1^{\circ}$ Ed. Ciudad de México: El manual moderno,SA.20 I7;1-2.

12. Silvestre Busto C. Práctica basada en la evidencia científica: elaboración y evaluación de guías de práctica clínica y protocolos. Rev. Enfermería en Anestesia, Reanimación y Terapia del Dolor. 2006; (I4):40-4.

13. Ariza C, Salazar AM. Lineamientos de investigación en Enfermería. Asociación Colombiana de Facultades de Enfermería; 20|4.10-5.

14. AguirreRD.Lainvestigaciónenenfermería enAmérica Latina200020 I 0. Revista Habanera de Ciencias Médicas. 20 I I: I 0(3):396-409.

15. Díaz HLP, Bueno RLS, Mejía RME, López L. Investigación de enfermería: una caracterización en dos publicaciones latinoamericanas. Av. enferm 201 I; 29(2):285-93.

16. Rojas M, Méndez R. Cómo enseñar e investigar. Un reto para la pedagogía universitaria. Educación 2013; I6(1):95-108.

17. Miguel Y, Peñaranda, R.Actitudes de los estudiantes de enfermería de la UNMSM hacia el curso de investigación y su relación con los factores personales e institucionales. [Tesis para optar el Grado de Magister en Educación en Enfermería]. Lima - Perú.UNMSM. 1994.

18. Cabrera-Enríquez JA, Cruzado-Mendoza C, Purizaca-Rosillo N, et al. Factores asociados con el nivel de conocimientos y la actitud hacia la investigación en estudiantes de medicina en Perú. RevPanam Salud Publica 20 I3; 33(3): 166-73. 


\section{Anexo}

Esta materia contribuye a estimular y apoyar a todos aquellos alumnos que quieran desarrollar trabajos de investigación en el Hospital. Por este motivo, nos interesa conocer tu opinión sobre algunos aspectos relacionados con tu experiencia, expectativas y necesidades para realizar trabajos de investigación. La Encuesta es anónima; agradecemos tu cooperación.

\begin{tabular}{|c|l|c|c|c|}
\hline Nro. & \multicolumn{1}{|c|}{ De acuerdo } & En desacuerdo & No estoy seguro \\
\hline $\mathbf{1}$ & $\begin{array}{l}\text { Recibí formación en la carrera que me ha permitido participar en } \\
\text { trabajos de investigación. }\end{array}$ & $\square 1$ & $\square 3$ \\
\hline 2 & $\begin{array}{l}\text { Considero que la participación en estudios de investigación es im- } \\
\text { portante para mi formación en esta etapa de mi carrera. }\end{array}$ & $\square 1$ & $\square 2$ & $\square 3$ \\
\hline 3 & $\begin{array}{l}\text { Entiendo que el desarrollo de trabajos de investigación podría me- } \\
\text { jorar mi labor diaria. }\end{array}$ & $\square 1$ & $\square 2$ \\
\hline
\end{tabular}

Marque todas las respuestas que considere necesarias.

\begin{tabular}{|c|l|c|}
\hline & Respecto de este curso: & \\
\hline 3 & Considero que va a mejorar mi formación académica & $\square 1$ \\
\hline 4 & Sólo debo realizarlo en forma obligatoria & $\square 1$ \\
\hline 5 & Considero que debomejorar/actualizar mis conocimientos sobre el desarrollo de trabajos de investigación & $\square 1$ \\
\hline 6 & Me interesan los temas que se desarrollarán & $\square 1$ \\
\hline
\end{tabular}

Indique su experiencia respecto a las siguientes frases o preguntas relacionadas con los trabajos de Investigación que ha desarrollado

\begin{tabular}{|c|l|c|c|c|}
\hline & & Nunca & Alguna vez & Más de una vez \\
\hline $\mathbf{7}$ & He participado en proyectos de investigación en su Hospital. & $\square 1$ & $\square 2$ & $\square 3$ \\
\hline $\mathbf{8}$ & He presentado trabajos de investigación en jornadas o en congresos como autor. & $\square 1$ & $\square 2$ & $\square 3$ \\
\hline $\mathbf{9}$ & $\begin{array}{l}\text { He participado como autor de trabajos de investigación o reportes de casos que han } \\
\text { sido publicados. }\end{array}$ & $\square 1$ & $\square 2$ & $\square 3$ \\
\hline 10 & He diseñado un estudio de investigación por iniciativa personal. & $\square 1$ & $\square 2$ & $\square 3$ \\
\hline
\end{tabular}

